\title{
Using CRISPRi to Repress Cell's Ability to Metabolize Glycerol and to Express LacZ
}

\author{
Guyin Chen ${ }^{1}$ and Shaun Spisak ${ }^{1}$
}

${ }^{1}$ Garrison Forest School, Waterville, ME, USA

\section{ABSTRACT}

CRISPR interference (CRISPRi) method is an effective way in disturbing a specific gene in the DNA by blocking transcription and therefore silencing a gene from expressing. When the catalytically dead Cas9 is coexpressed with a guide RNA, it generates a DNA recognition complex that can target a specific gene. The project focuses on manipulating the genes of DNA to repress certain gene expressions. The first assay involves repressing the genes that allow cells to grow in glycerol, and the second assay targets the cell's ability to break down lacZ.

\section{Summary}

CRISPR interference (CRISPRi) method is an effective way in disturbing a specific gene in the DNA by block transcription and therefore silencing a gene from expressing. This method is developed based on Cas9, an RNA-guided DNA endonuclease. When the catalytically dead Cas9 is coexpressed with a guide RNA, it generates a DNA recognition complex that can target a specific gene. The project focuses on manipulating the genes of DNA to repress certain gene expressions. The goal is to manipulate the genes in the DNA so that specific expressions are repressed. We started with making the sgRNA that will be used to guide dCas9 to the specific location on the DNA, which can be used to repress the target genes. The first assay involves repressing the genes that allow cells to grow in glycerol, and by repressing the gene using sgRNA and dCas9, the cell should be unable to grow in glycerol. The second assay targets the cell's ability to break down lacZ by repressing the gene that encodes the protein lacZ, which breaks down lactose.

\section{Introduction}

Genome editing is a new invention that has proven to be effective in preventing and treating diseases, and more research is done on gene editing nowadays to discover the important uses of it ("What Are Genomes," n.d.). CRISPR (Cluster of Regularly Interspaced Palindromic Repeats), was initially discovered in the E. Coli genome in 1988. It provides an immunity against viral infection, and it was viewed as a potential for gene editing. CRISPR systems use base-pairing RNAs to target foreign DNA, and the simplest one is the type II CRISPR system from Streptococcus pyogenes, and it has one endonuclease Cas9 and two types of RNA. When bacteria captures a segment of the DNA from an invading virus, it uses the segment to create a CRISPR array, which allows bacteria to encode memory of the virus. Thus, when facing the attack from a similar virus, the bacteria can use CRISPR arrays to target the viruses' DNA and use Cas9 to cut the DNA apart, thus disabling the virus from invading ("What Are Genomes," n.d.).

There are two different types of gene editing methods that arose from CRISPR: CRISPRa (CRISPR activation), and CRISPRi(CRISPR interference). In both systems, Cas9 is used to interact with transcriptional effectors ("CRISPRa and CRISPRi," n.d.). CRISPRi specifically uses dCas9, which does not have the ability to cut DNA but can still target a specific gene location, to repress gene expression ("CRISPRa and CRISPRi," n.d.). CRISPRi gets in the way of the 
protein that normally transcribes DNA into RNA and prevents the protein from transcribing. Thus, the catalytically dead form of the Cas9 gene (dCas9) can be used to silent genes via transcriptional repression, which is guided by RNA (Qi, Larson, \& Gilbert, 2013, p.2). In order to target a specific gene, a single specific guide RNA (sgRNA) must be developed, and these sgRNA contains three regions: a base-pairing region that is $20 \mathrm{nt}$, a dCas9-binding handle that is $42 \mathrm{nt}$, and a terminator region that is $40 \mathrm{nt}$ (Simmons, 2019). In CRISPRa, the RNA, acts as a guide for Cas9 enzyme, finds the specific part of the DNA and allows Cas9 to cut DNA at the targeted location. After that, a piece of genetic material can be either cut or replaced by another segment of DNA ("What Are Genomes," n.d.). The project mainly focuses on CRIAPRi, which has been proven to be able to repress targeted genes in cells without significant off-target effects, and repress multiple target genes simultaneously with reversible effects ("What Are Genomes," n.d.).

The lab focuses on manipulating CRISPRi to repress a cell's ability to grow in glycerol and cell's ability to break down lactose. By manipulating the genes of DNA, we are able to observe whether these cell expressions are expressed or oppressed in the end. We started with manipulating the sgRNA, so that it will be able to guide dCas9 correctly to the targeted area. We examined about 20 nucleotides that correspond with nucleotides in DNA, and by changing the sequence of nucleotides in the sgRNA and pairing it up with the correct amount of dCas9, we are able to repress specific genes from expressing in the cell.

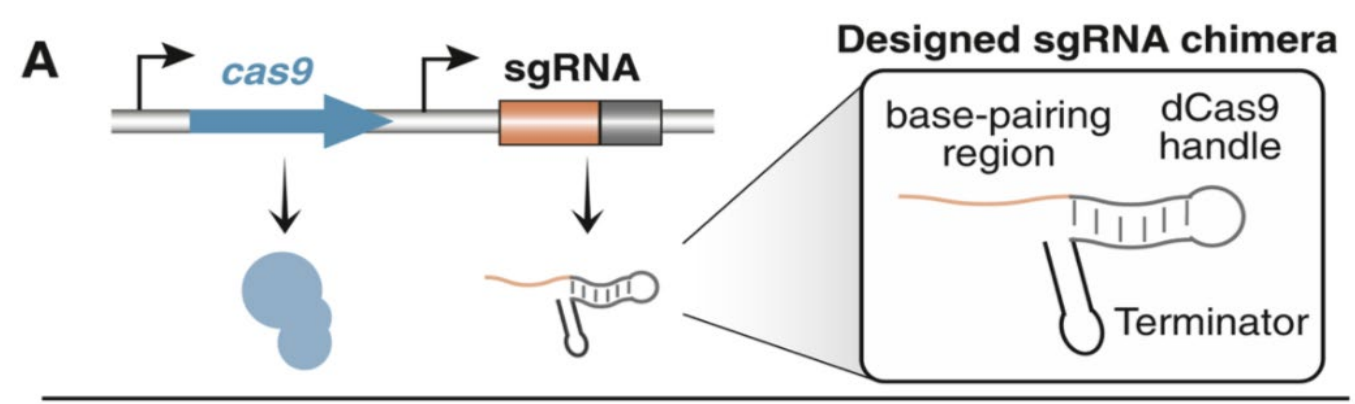

B
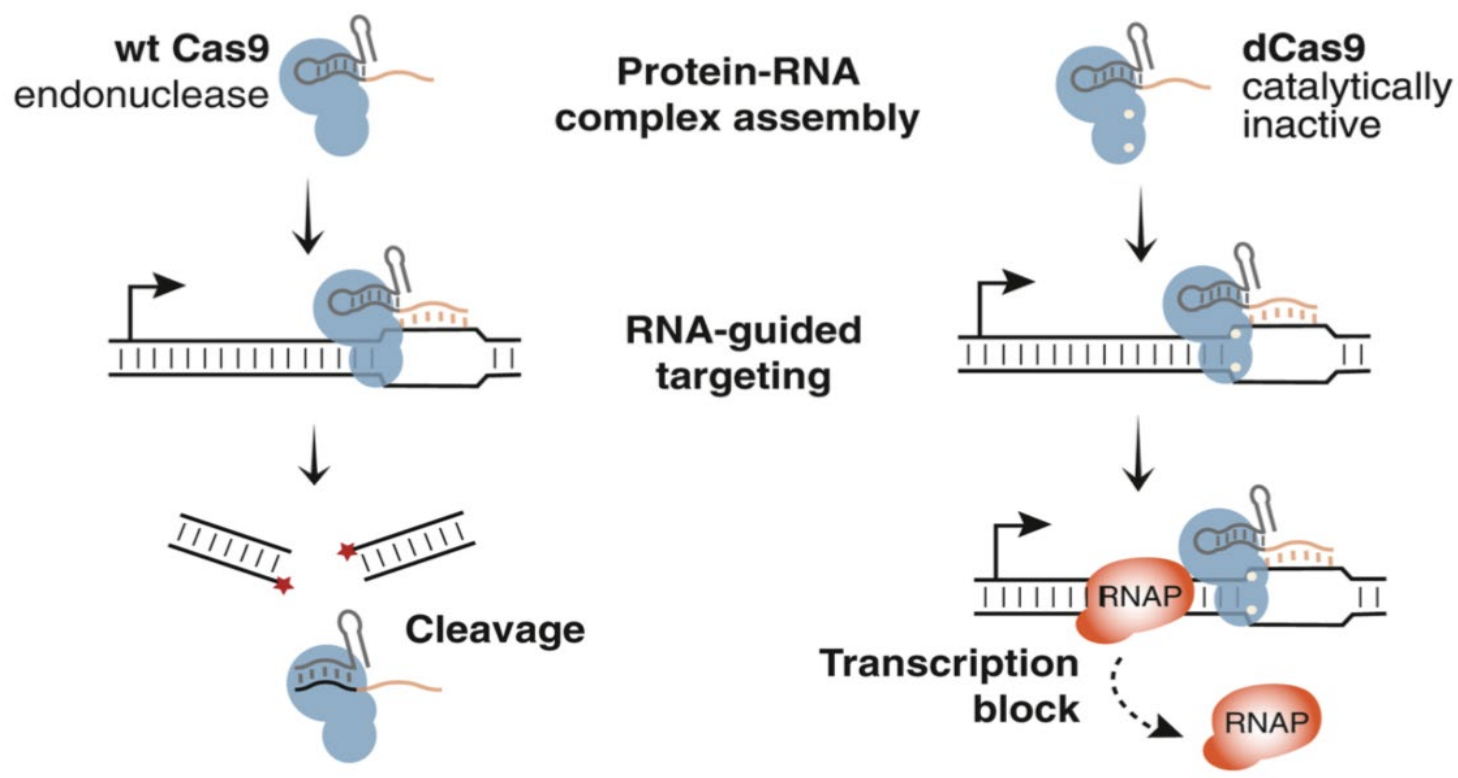

Figure 1: Design of CRISPRi (Qi, Larson, \& Gilbert, 2013, p.13) 
(A) Shows the design of sgRNA chimera, which has Cas9 and a sgRNA. This sgRNA consists of a base-pairing region, which binds to the targeted area of the DNA, a dCas9 handle, which binds to dCas9, and a terminator that controls where to end on the DNA.

(B) This sgRNA can bind with either Cas9 or dCas9, which is catalytically inactive., When sgRNA binds with Cas9, it can cleavage the DNA. When sgRNA binds with dCas9, it blocks the transcription of DNA at the targeted location.

\section{Results}

\section{CRISPRi Can Effectively Repress Cells’ Ability to Grow in Glycerol}

The purpose of this experiment is to use CRISPRi to repress cells' ability to grow in glycerol. There are two tubes that serve as control groups, and the control groups are expressing a guide RNA, but the sgRNA is not targeting the gene responsible for metabolizing glycerol. The other two tubes have sgRNA targeting the genes responsible for metabolizing glycerol, and each of the two sets of tubes has a low concentration of dCas 9 and a high concentration of dCas9. Since sgRNA has to target the correct location for repressing metabolization of glycerol and a high concentration of dCas9 will increase the likelihood of repressing the expression, the hypothesis is that there should be little or no growth in the on target tube with a high concentration of dCas9. Tubes are put in the shakers at $37^{\circ} \mathrm{C}$, which is considered to be the optimum growth temperature for E.Coli cells, and the growth of the cell is recorded at $0 \mathrm{hr}, 6 \mathrm{hr}$, and every hour starting from $18 \mathrm{hr}$ until $28 \mathrm{hr}$. The experiment is repeated three times, and the growth for all three trials are recorded and the mean value is taken for each time point.

In this study, the off-target tube with the low concentration of dCas9 has the most amount of growth while the on-target tube with the high concentration of dCas9 has the least amount of growth. This proves that the CRISPRi system, with the sgRNA targeting the correct location in the gene, can repress the ability for cells to metabolize glycerol dramatically, with the growth of less than 0.05 OD 600. The amount of dCas9 has an influence on the effectiveness of the CRISPRi system. The study suggests that the high level of dCas 9 with on target sgRNA corresponds with minimal amount of growth $(<0.025$ OD 600$)$ while the low level of dCas9 has a higher amount of growth $(<0.05$ OD 600).

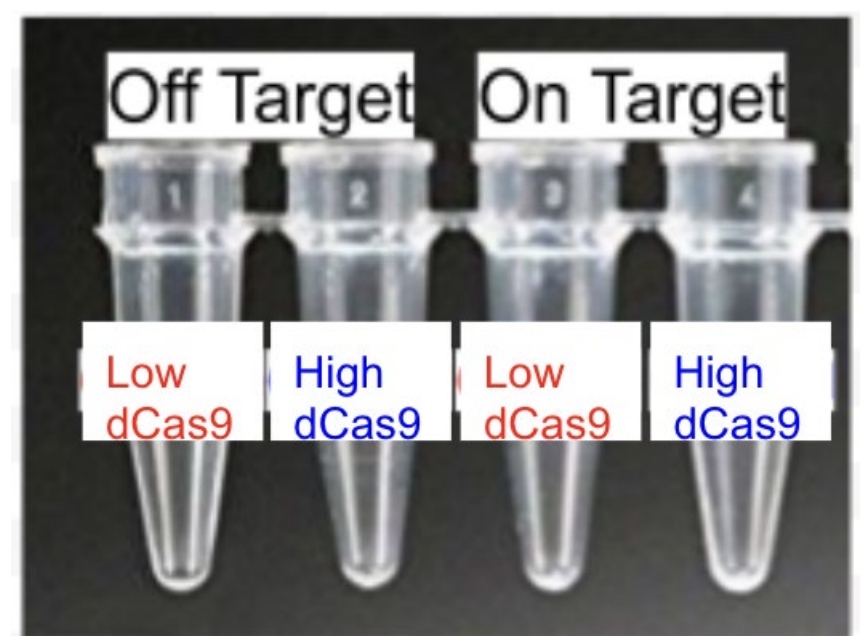

Figure 2: The Setup of Glycerol Repression Assay

There are two off-target tubes that serve as control groups. The control groups have the sgRNA targeting the incorrect gene in each of them, and they each have a high and a low concentration of dCas9. The other two groups are on-target 
groups that have the sgRNA targeting the correct location in them, and each tube also has a low and a high concentration of dCas9.

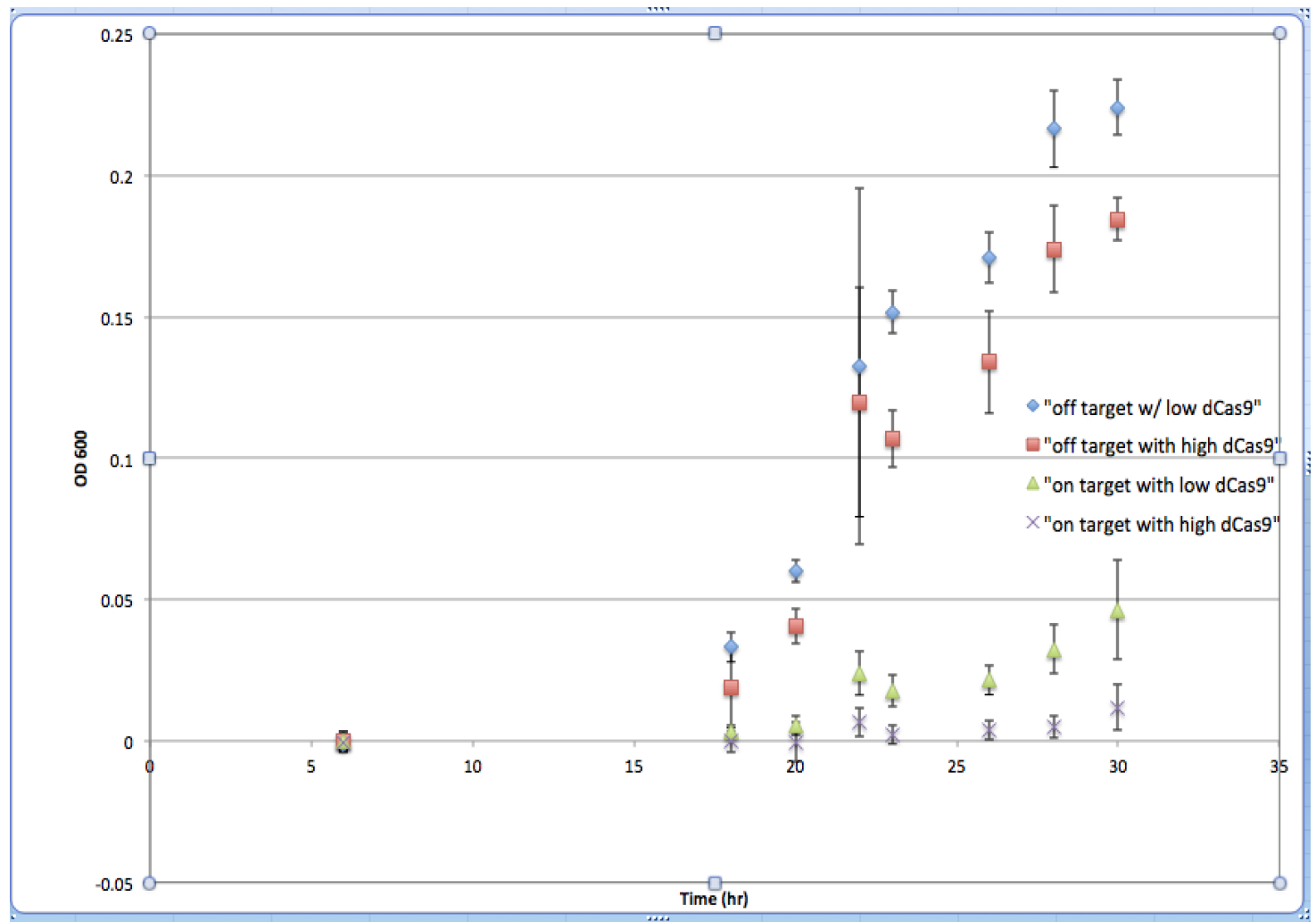

Figure 3: Graphical result for Glycerol Assay

According to the graph, the off-target tube with low concentration of dCas9 has the most amount of growth, followed by the off-target tube with high dCas9 level. The two on- target tubes have significantly less growth than off-target tubes. The on-target with low dCas9 level has more growth than the on-target tube with high dCas9 level. This indicates that with the sgRNA targets the correct location, the higher concentration of dCas9 produces the most effective repression of cell growth in glycerol.

\section{CRISPRi Can Repress the Genes for Breaking Down Lactose in Cells}

In this experiment, we explored CRISPRi's ability to repress genes responsible for breaking down lactose in cells by observing how much LacZ is present in the solution. We used ONPG, which breaks down into a yellow color, making the result easier to visualize since we can determine the concentration of yellow color in the solution. Thus, by comparing how yellow the solution is, we can determine how much of the LacZ gene is being repressed. There are four tubes with two off-target tubes and two on-target tubes, and each set of tubes contains a minimal amount of dCas9 and a maximum amount of dCas9. The hypothesis is that the on-target tube with the maximum amount of dCas9 will have the least color. We conducted our experiment by preparing solutions to react for $15 \mathrm{~min}, 30 \mathrm{~min}$, and $60 \mathrm{~min}$. Every time after the reaction runs in completion, we add a stop solution to the tubes and monitor the color of the solutions using a spectrophotometer. 
For the 15 min reactions, there is no clear yellow color detected, and that might be due to the lack of reaction time. When we looked at the $30 \mathrm{~min}$ ones, some tubes looked a little yellowish but mostly clear. In the 60 min one, the solutions that are off-target looked really yellow, while the on-target solutions looked mostly clear. We repeated the experiment three times and collected data for each trial to ensure that the result is statistically significant and expelling the possibility of confounding variables. When we measured the concentration on the spectrophotometer, the result corresponds with the hypothesis well: The concentration of the on-target solution with high dCas 9 concentration is essentially 0 for both $30 \mathrm{~min}$ and $60 \mathrm{~min}$ trial, the on target with low dCas9 has a minimal growth (<10 Miller Unit). For the trials with off target sgRNA, both $30 \mathrm{~min}$ and $60 \mathrm{~min}$ trials have a large amount of growth (> 150 Miller Unit). This data proves that the correctly targeted sgRNA combining with a high concentration of dCas 9 can successfully repress the expression of lacZ.

\section{OFF TARGET ON TARGET}

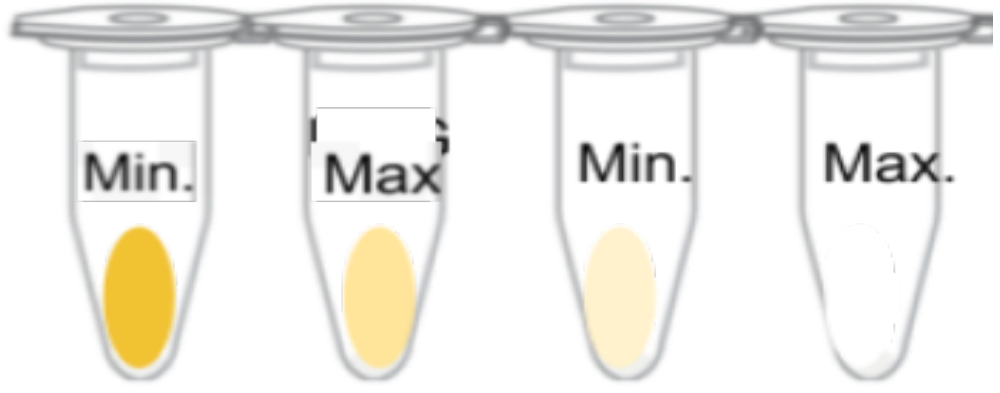

Figure 4: Setup for $\boldsymbol{\beta}$-Galactosidase Expression Assay

There are two off-target tubes that serve as control groups. The control groups have the sgRNA targeting the incorrect gene in each of them, and they each have a high and a low concentration of dCas9. The other two groups are on-target groups that have the sgRNA targeting the correct location in them, and each tube also has a low and a high concentration of dCas9.

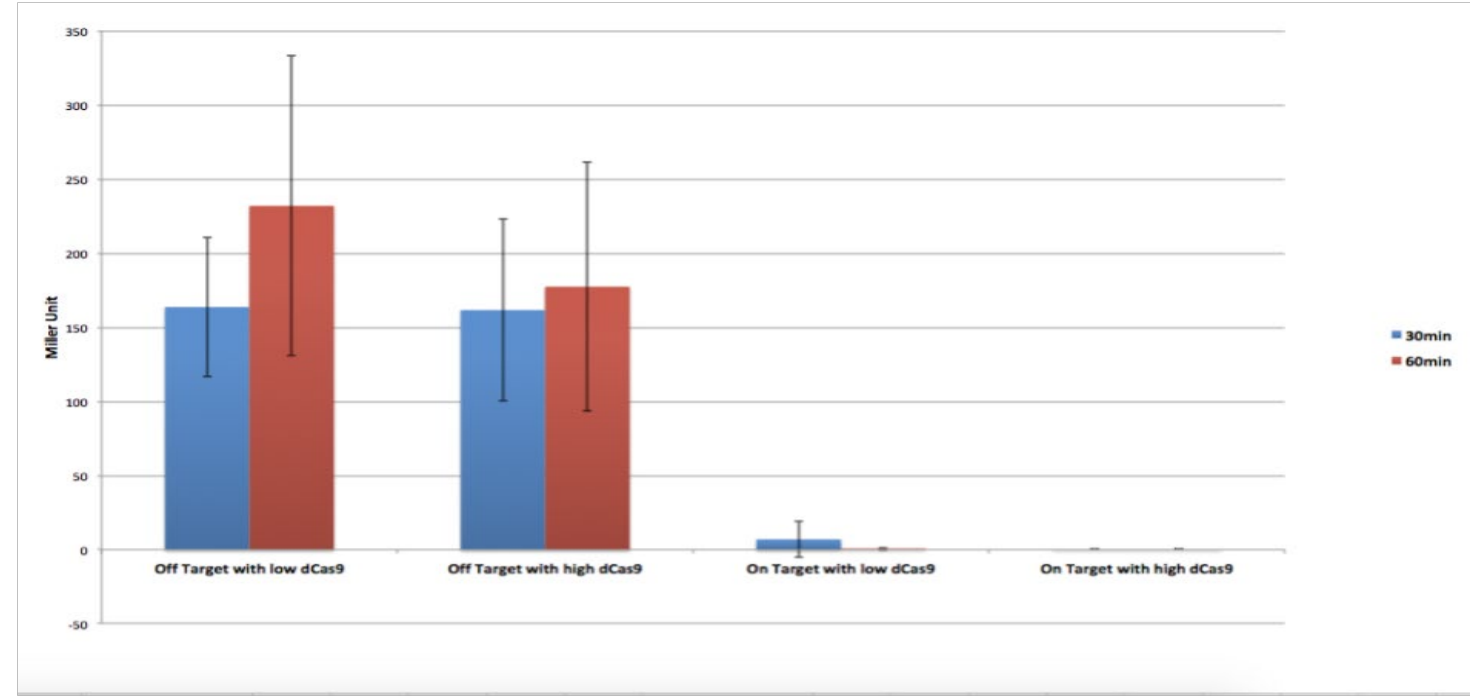

Figure 5: Graphical Result for $\boldsymbol{\beta}$-Galactosidase Expression Assay 
The graph shows that the off-target tube with low dCas9 concentration has the most amount of growth in both 30 min and 60 min trials. The off-target with high dCas 9 concentration also has a large amount of growth. By comparison, the on target tubes all have minimal growth with the tube containing low dCas9 level has slightly more growth than on-target with high dCas9 level.

\section{Experimental Proceedures}

\section{Polymerase Chain Reaction (NIH, 2015)}

PCR is used to produce copies of a segment of DNA. In order to proceed PCR, a DNA template to be copied, a primer that is designed to bind to either side of the section of DNA, a DNA nucleotide base, a polymerase enzyme, and a buffer are needed to proceed the reaction. First, a sample is heated so the DNA denatures and separates into two pieces of single-stranded DNA. Then, the temperature is lowered to enable the DNA primers to attach to the template DNA. Lastly, the temperature is raised again, and the new strand of DNA is made by the Taq polymerase enzyme. After that, each strand can be used to create new copies, and the cycle is repeated 30 to 40 times, making more than a billion copies of the original DNA segment.

\section{Agarose Gel Electrophoresis (“Agarose Gel Electrophoresis”, 2018)}

Agarose Gel electrophoresis is used to separate DNA by size for visualization and purification, and shorter DNA will move through the gel more quickly than the long ones, which allows you to visualize the approximate length of DNA fragments along a DNA ladder (a sample of DNA fragments with known lengths). The procedure is conducted by measuring $1 \mathrm{~g}$ of agarose and mixing the agarose with $100 \mathrm{~mL} 1 \mathrm{xTAE}$ in a flask and microwaving it for 1-3 minutes until the agarose is fully dissolved. After the solution cools down, pour the agarose into a gel tray with a well comb and load a DNA sample to each well along with a sample DNA ladder. Then, fill the gel box with more 1xTAE until the gel is fully covered and start running the gel at $80-150 \mathrm{~V}$ until the dye line is approximately $75-80 \%$ of the way down the gel. Then, use UV light to visualize the DNA fragments.

\section{Invitrogen Gel Extraction (PureLink® Quick, 2011)}

Invitrogen Gel Extraction is used to purify DNA. First, equilibrate a water bath to $50{ }^{\circ} \mathrm{C}$ and excise a minimal area of gel containing the DNA fragment and weigh it. Then, add Gel Solubilization Buffer to the gel and place the tube with the gel into the $50^{\circ} \mathrm{C}$ water bath. Incubate the tube for 10 minutes and invert the tube to ensure gel dissolution. Then, purify the DNA using a centrifuge by loading the dissolved gel into a wash tube and adding a wash buffer. After spinning the column for 1 minute, discard the flow-through and place the column into the wash tube. Repeat the process two times, and store the purified DNA at $4^{\circ} \mathrm{C}$.

\section{Electroporation (“Electroporation Protocol,” n.d.)}

Electroporation is used to introduce DNA, in our case, the plasmid, into cells using a pulse of electricity to briefly open the pores in cell membranes. First prepare a culture tube and place SOC recovery medium in a $37^{\circ} \mathrm{C}$ water bath, and then place electroporation cuvettes and microcentrifuge tubes on ice. Thaw NEB Turbo Electrocompetent cells on ice and mix cells, and transfer $25 \mathrm{ul}$ of cells to a chilled microcentrifuge tube and add $1 \mathrm{ul}$ of DNA solution. Transfer the mix into a chilled civette and electroporate the cuvette using $2.1 \mathrm{kV}$. Then, immediately add $975 \mathrm{ul}$ of 37 ${ }^{\circ} \mathrm{C} \mathrm{SOC}$ to the cuvette and mix it gently before transfering to a culture tube. Shake the tube at $37^{\circ} \mathrm{C}$ for an hour and 
dilute the cell as appropriate then spread 100-200 ul onto a pre-warmed selective plate and incubate plates 8 hours to overnight at $37^{\circ} \mathrm{C}$.

\section{Sanger DNA Sequencing ("Sanger DNA Sequencing," n.d.)}

Sanger sequencing is used to determine the nucleotide sequence of DNA. DNA polymerase adds dNTPs to a growing DNA strand by catalyzing the formation of a phosphodiester bond between the free 3'-OH group of the last nucleotide and the 5'-phosphate of the next. Then, mix a low ratio of chain-terminating ddNTPs in the normal dNTPs. Then, the chain-terminated oligonucleotides are separated by size via gel electrophoresis, in which samples are loaded into one end of a gel matrix, and an electric current is applied. The speed these oligonucleotides move will be determined by the size of the DNA sample. The computer reads each bank of the capillary gel in order using fluorescence to call the identity of terminal ddNTP. Since each fluorescence emits different light, the identity of the terminal ddNTP can be discovered. Then, an output called chromatogram is shown, which shows the fluorescent peak of each nucleotide along the length of the template DNA.

\section{Recovering Plasmid DNA from Bacterial Culture ("Recovery Plasmid," 2018)}

Plasmid Miniprep is used to purify plasmid DNA from bacterial culture. First, grow an overnight culture of bacteria then centrifuge the culture to pellet the bacteria. Then, remove the supernatant and resuspend bacteria in a buffer and add a denaturing solution to the resuspended bacteria, which causes bacteria to lyse and release their contents in the solution. Then, pellet the proteins and genomic DNA by centrifugation and remove the plasmid-containing supernatant. After that, add either ethanol or isopropyl to precipitate the plasmid DNA and spin the DNA to get the precipitate. Then, wash the pellet with $70 \%$ ethanol to remove any excess salt and resuspend DNA pellet.

\section{Acknowledgments}

We thank Dr. Ostermeier for allowing this experiment to happen and Shaun Spisak for guiding the experiment. We also thank the support from Mrs. Perry, Dr. Blair, and Ms. O.

\section{Discussion}

The study demonstrates the ability of the CRISPRi system to repress the metabolism of cells in glycerol with the correct binding of sgRNA and level of dCas9. By manipulating CRISPRi to repress cells' ability to grow in glycerol, the lab can further its research on the potential of making a "switch protein" that allows us to manually control when to turn on and off the cells' ability to grow in glycerol. By turning off the glycerol metabolism system when there's no glycerol present, the cells can save energy, which can be used to do further work. Also, the switch protein can manipulate the level of glycerol metabolism by potentially changing the level of dCas9, which allows further control of the cell. Similarly, a switch protein for controlling the breakdown of lactose can also be made by switching on and off the cells' ability to breakdown lactose and control how much lactose would be broken down. Thus, the result from the study can potentially be used to create switch proteins to manipulate the specific ability of the cell to make the cell function more efficiently. 


\section{References}

Agarose Gel Electrophoresis. (2018, February 20). Retrieved May 7, 2020, from https://www.addgene.org/protocols/gel-electrophoresis/

CRISPRa and CRISPRi: Gene Expression Modulation. (n.d.). Retrieved May 7, 2020, from https://www.origene.com/products/gene-expression/crispr-cas9/crispra-crispri

Electroporation Protocol (C2986). (n.d.). Retrieved May 7, 2020, from https://www.neb.com/protocols/0001/01/01/electroporation-protocol-c2986

Polymerase Chain Reaction (PCR) Fact Sheet. (2015, June 16). Retrieved May 6, 2020, from https://www.genome.gov/about-genomics/fact-sheets/Polymerase-Chain-Reaction-Fact-Sheet

PureLink® Quick Gel Extraction Kit. (2011, March 7). Retrieved May 8, 2020, from https://assets.thermofisher.com/TFS-Assets/LSG/manuals/purelink_quick_gel_extraction_kit_qrc.pdf

Qi, L. S., Larson, M. H., \& Gilbert, L. A. (2013, May). Repurposing CRISPR as an RNA-Guided Platform for Sequence- Specific Control of Gene Expression. PMC.

Recovering Plasmid DNA from Bacterial Culture. (2018, February 8). Retrieved May 7, 2020, from https://www.addgene.org/protocols/purify-plasmid-dna/

Sanger DNA Sequencing Steps and Method. (n.d.). Retrieved May 7, 2020, from https://www.sigmaaldrich.com/technical-documents/articles/biology/sanger-sequencing.html

Simmons, H. (2019, February 26). CRISPR Interference (CRISPRi) for Gene Suppression. Retrieved May 6, 2020, from https://www.news-medical.net/life-sciences/CRISPR-Interference-(CRISPRi)-for-Gene-Suppression.aspx

What are genome editing and CRISPR-Cas9? (n.d.). Retrieved from https:/ghr.nlm.nih.gov/primer/genomicresearch/genomeediting 